Dans son papier de position, la FMH exige l'interdiction de toute publicité, de toute promotion et de tout parrainage des produits du tabac afin de permettre notamment aux enfants et aux adolescents d'envisager un avenir sans cigarette. Le présent article souligne la nécessité d'instaurer une interdiction généralisée pour protéger les enfants et les adolescents, cibles préférées des publicitaires de l'industrie du tabac. Pendant combien de temps voulons-nous rester les bras croisés?

Dr Christine Romann, membre du Comité central de la FMH, responsable du département Promotion de la santé et prévention

\title{
Publicité en faveur du tabac: l'attirance d'une cigarette cachée
}

\section{Iris Leu}

Collaboratrice scientifique, division Promotion de la santé et prévention

La Convention-cadre de l'OMS pour la lutte antitabac (CCLAT) énonce plusieurs mesures dont notamment une interdiction généralisée de la publicité pour le tabac. La Suisse a certes signé cette convention mais sans pour l'heure l'avoir mise en œuvre. La synthèse de l'étude de l'observatoire permet de mieux cerner les stratégies de marketing pour les produits du tabac, omniprésentes en Suisse en comparaison à d'autres pays.

Alors que les stratégies marketing de l'industrie du tabac se déclinent en trois catégories principales, à savoir la publicité, la promotion et le parrainage, la Convention-cadre de l'OMS pour la lutte antitabac (CCLAT) constitue le premier traité mondial de santé publique élaboré en réponse à la mondialisation de l'épidémie de tabagisme. Elle édicte des mesures efficaces comme l'interdiction de la publicité pour le tabac, de la promotion et du parrainage. Cependant, pour que l'interdiction porte ses fruits, elle doit inclure toutes les formes de marketing, ce que fait la définition de la CCLAT:

- Publicité en faveur du tabac et promotion du tabac: toute forme de communication, recommandation ou action commerciale ayant pour but, effet ou effet vraisemblable de promouvoir directement ou indirectement un produit du tabac ou l'usage du tabac.

- Parrainage du tabac: toute forme de contribution à tout événement, activité ou personne, ayant pour but, effet ou effet vraisemblable de promouvoir directement ou indirectement un produit du tabac ou l'usage du tabac.

La Suisse a signé la CCLAT en 2004 mais elle est aussi un des rares pays européens à ne pas l'avoir ratifiée. Sa législation actuelle en matière de publicité en faveur du tabac est lacunaire et diffère d'un canton à l'autre. Certains cantons adoptent une législation beaucoup plus stricte que d'autres en matière de publicité, de pro-

L'interdiction globale de la publicité dans les pays riches permet de diminuer en moyenne de $7 \%$ la consommation de tabac.

motion et de parrainage [1]. Cette situation laisse une marge de manœuvre importante à l'industrie du tabac. C'est aussi ce qui explique l'omniprésence du marketing en Suisse en comparaison avec la majeure partie des pays européens. Ici, seule la publicité à la radio et à la télévision est interdite.

Il est prouvé que la publicité pour les produits du tabac a un impact important sur la consommation et, avec

\section{Etude complète}

Vous trouverez l'étude complète en version en ligne: www.observatoire-marketing-tabac.ch/ $\rightarrow$ Présentation $\rightarrow$ Matériel $\rightarrow$ Une brochure de présentation des résultats de I'Observatoire 
9000 décès chaque année en Suisse, le tabagisme reste la première cause évitable de mortalité. C'est la raison pour laquelle l'Observatoire des stratégies marketing pour les produits du tabac a été lancé.

L'industrie du tabac déploie des stratégies très subtiles pour que la promotion de ses produits soit à la fois très visible par son public cible (les jeunes et les fumeurs) et relativement invisible par le reste de la population. Elle agit dans les bars, boîtes de nuit et cinémas, dans la presse, aux points de vente, lors de festivals ou de manifestations privées qu'elle organise ou parraine.

Les observations empiriques montrent que l'industrie du tabac cible ses stratégies clairement sur les jeunes, notamment par le langage utilisé, le graphisme, les photos et les lieux de la publicité. La cigarette est présentée en lien avec des systèmes de référence (fête, goût du risque, flirt, vacances, etc.) qui évoquent des associations positives chez les jeunes. Les jeunes sont réceptifs aux messages publicitaires, comme le démontrent 18 études à long terme [1] menées auprès de 27000 jeunes non-fumeurs âgés entre 8 et 18 ans et démontrant que le fait d'être confronté à la publicité pour le tabac augmente la probabilité de commencer à fumer.
L'Office fédéral de la santé publique (OFSP) estime à 18000 exemplaires le nombre total d'automates à cigarettes présents en Suisse, alors que dans plusieurs pays européens (France, Royaume-Uni, Irlande et Grèce) ces mêmes automates ont été interdits.

L'étude passe en revue différents éléments de publicité directe dans le quotidien des jeunes. Pendant une journée de week-end avec des activités habituelles et une sortie le soir, les jeunes en Suisse romande sont confrontés en moyenne à 68 incitatifs favorables à la consommation de tabac.

Dans notre pays, les marques de tabac disposent de sites Internet leur permettant de faire de la publicité et de publier des informations concernant leurs activités. Il faut souligner que parmi les 22 sites recensés pour l'étude, 12 sont accessibles aux mineurs.

La publicité en faveur du tabac est également très présente sur les réseaux sociaux. L'industrie du tabac se sert de ces plates-formes de communication pour faire la publicité et la promotion de ses produits. Les internautes agissent ensuite comme de véritables porteparoles en transmettant les messages publicitaires de leur marque préférée. Le phénomène des Smoking

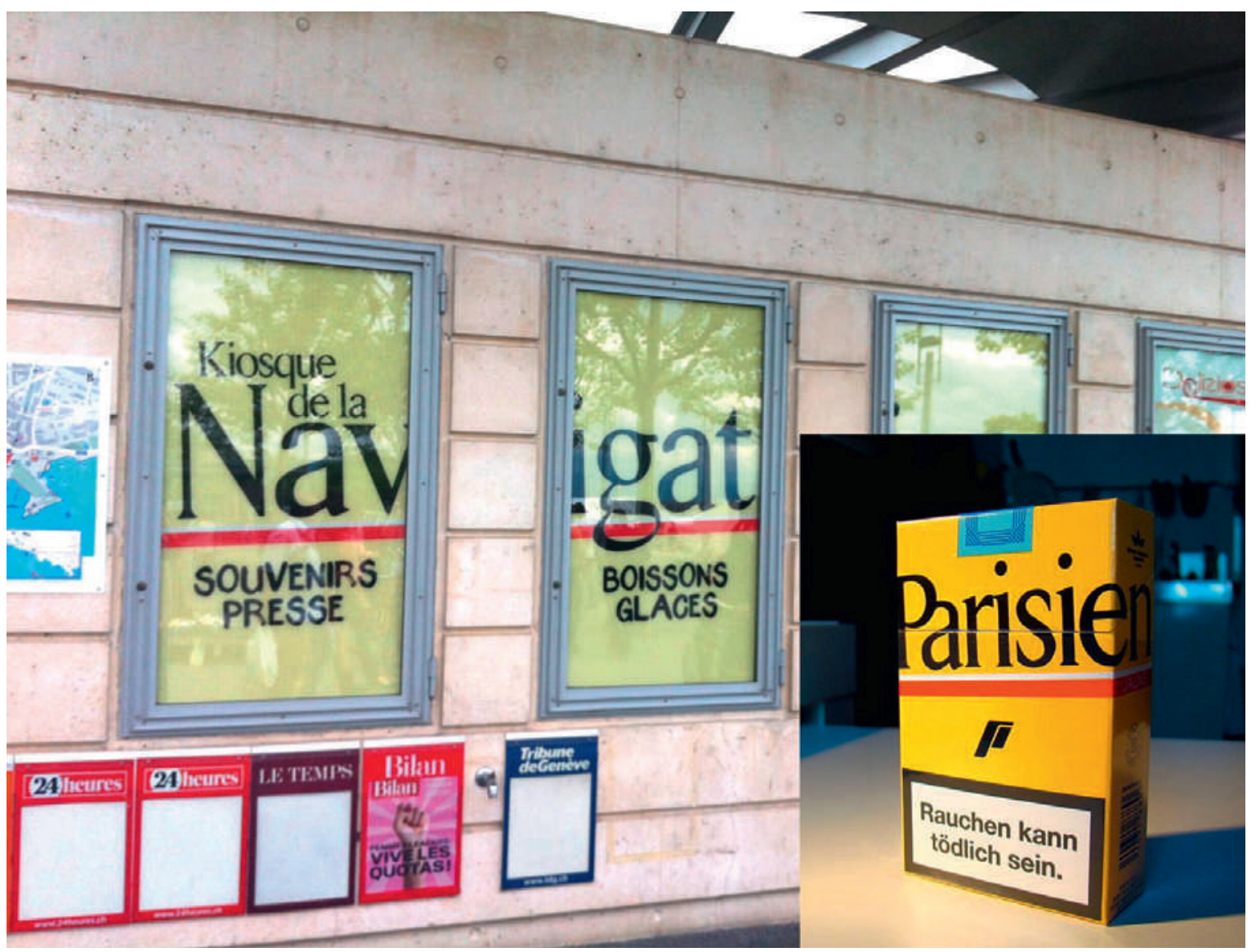

Illustration 1: Evocation de la marque Parisienne - un exemple de publicité déguisée. 
Reviews sur Youtube est en ce sens particulièrement intéressant. Dans ces vidéos, les consommateurs, souvent très jeunes, «dégustent» les produits et se mettent en scène en train de fumer, ce qui ne fait que renforcer le caractère "cool» de la cigarette.

Les tactiques de marketing sont extrêmement novatrices. La publicité ne se limite pas à des formes classiques (campagne avec des affiches ou publicité dans la presse) mais elle mise sur le marketing expérientiel, le marketing personnalisé, le marketing participatif et la publicité déguisée.

L'objectif du marketing expérientiel consiste à proposer une expérience unique et à mettre en éveil les sens du consommateur (les papilles avec la consommation de plats festifs, la consommation d'alcool; les émotions positives liées à la nature de l'événement, etc.) ou à mettre en scène la consommation de tabac dans le cadre d'expériences à fort caractère émotionnel (prendre des risques, défier la norme, etc.). Cela encourage à consommer du tabac. Le marketing personnalisé vise à toucher le client de manière directe et personnelle, en donnant la sensation au consommateur d'être unique et privilégié dans sa relation à la marque. Le marketing participatif est une approche consistant à donner au consommateur un rôle dans le développement d'un produit ou dans la diffusion de messages auprès d'autres consommateurs; le principe étant qu'un consommateur impliqué sera bien plus écouté par son entourage que si la marque prend la parole elle-même. La publicité déguisée vise à employer la ligne graphique d'une marque (police, logo, couleurs, etc.) sans que son nom apparaisse. Ceci contribue à créer un effet de familiarité en rendant le produit plus désirable chez les consommateurs (ill. 1).

Ce genre de méthodes touchent particulièrement les jeunes consommateurs, elles maintiennent et/ou augmentent la consommation de tabac des fumeurs, découragent ceux qui souhaiteraient s'arrêter et encouragent les anciens fumeurs à recommencer.

Les cigarettes électroniques suscitent toujours plus d'attention. Compte tenu du fait qu'elles ne sont, à ce jour, pas assimilées à un produit du tabac, la publicité pour ces produits ne fait l'objet d'aucune réglemen- tation. Les observations ont démontré que la majorité des acheteurs de cigarettes électroniques sont des fumeurs adultes qui souhaitent réduire ou arrêter leur consommation de cigarettes classiques.

Une loi sur les produits du tabac a été élaborée dans la foulée de la révision de la loi sur les denrées alimentaires (LDAl) et mise en consultation à l'été 2014.

L'avant-projet du Conseil fédéral contient plusieurs améliorations par rapport à la loi actuelle. Il veut par exemple interdire la publicité pour le tabac dans la presse écrite, sur le mobilier urbain et dans les cinémas. Du point de vue de la prévention, l'interdiction de publicité devrait cependant s'appliquer à tous les domaines afin de produire l'effet escompté.

\section{L'industrie du tabac se sert des plates-formes de communication pour faire la publicité et la promotion de ses produits.}

De nombreux pays ont déjà interdit toute forme de publicité en faveur du tabac, de promotion et de parrainage. Ces mesures sont efficaces et ont un impact sur la consommation. L'interdiction globale de la publicité dans les pays riches permet de diminuer en moyenne de $7 \%$ la consommation de tabac. Pour qu'elle soit efficace, l'interdiction doit être globale. En effet, les interdictions partielles sont inutiles car l'argent qui ne peut plus être dépensé dans un secteur déterminé est réalloué là où la publicité est toujours autorisée.

\section{Références}

1 Cf. tableau de la législation actuelle concernant la publicité pour le tabac, la promotion et le parrainage, de l'Observatoire des stratégies marketing pour les produits du tabac en Suisse romande, 2013-3014, page 9.

2 Lovato $\mathrm{C}$ et al. Impact of tobacco advertising and promotion on increasing adolescent smoking behaviours. Cochrane Database of Systematic Reviews. 2011;5(10).

L'Observatoire des stratégies marketing pour les produits du tabac a été financé par le Fonds de prévention du tabagisme et a été réalisé par le CIPRET-Vaud en collaboration avec Addiction Suisse et le CIPRET Fribourg. 